\title{
An Automated Sensing System for Steel Bridge Inspection Using GMR Sensor Array and Magnetic Wheels of Climbing Robot
}

\author{
Rui Wang and Youhei Kawamura \\ Department of Intelligent Interaction Technologies, University of Tsukuba, Tsukuba, Ibaraki 305-8573, Japan \\ Correspondence should be addressed to Rui Wang; wangrui315216@yahoo.co.jp
}

Received 2 June 2015; Revised 12 October 2015; Accepted 20 October 2015

Academic Editor: Paddy French

Copyright ( 2016 R. Wang and Y. Kawamura. This is an open access article distributed under the Creative Commons Attribution License, which permits unrestricted use, distribution, and reproduction in any medium, provided the original work is properly cited.

\begin{abstract}
Corrosion is one of the main causes of deterioration of steel bridges. It may cause metal loss and fatigue cracks in the steel components, which would lead to the collapse of steel bridges. This paper presents an automated sensing system to detect corrosion, crack, and other kinds of defects using a GMR (Giant Magnetoresistance) sensor array. Defects will change the relative permeability and electrical conductivity of the material. As a result, magnetic field density generated by ferromagnetic material and the magnetic wheels will be changed. The defects are able to be detected by using GMR sensor array to measure the changes of magnetic flux density. In this study, magnetic wheels are used not only as the adhesion device of the robot, but also as an excitation source to provide the exciting magnetic field for the sensing system. Furthermore, compared to the eddy current method and the MFL (magnetic flux leakage) method, this sensing system suppresses the noise from lift-off value fluctuation by measuring the vertical component of induced magnetic field that is perpendicular to the surface of the specimen in the corrosion inspection. Simulations and experimental results validated the feasibility of the system for the automated defect inspection.
\end{abstract}

\section{Introduction}

Steel bridges represent a very important investment in the transportation network, which support a nation's economy and traffic. They are widely used for infrastructure such as railways and roads. Steel bridges are subjected to serious deterioration due to natural aging and load spectra. In America, most of steel bridges on the US Interstate Highway System were constructed after World War II from 1950 to 1980. Over 40000 steel bridges are structurally deficient and over 35000 are functionally obsolete [1]. In Japan, the construction of the steel bridges has been slowed down but the number of steel bridges that have been constructed more than 50 years ago is increasing rapidly. It has been reported that in 20 years more than half of the existing steel bridges are predicted to be over 50 years old [2]. Figure 1 shows the number of steel bridges constructed in Japan. It is obvious that steel bridges in Japan face a deterioration problem. Periodical inspection of steel bridges is essential for the long-term safety of public infrastructures.
Corrosion is one of the most important reasons for the deterioration of steel bridges. It is a gradual deterioration process of metals by chemical or electrochemical reactions in their environments. As time goes on, old steel bridges suffer from corrosion problems, which seriously threaten the safety of the steel bridge. In America, some estimates have indicated that the cost of metal corrosion damage to highway bridges alone could reach $\$ 6-\$ 10$ billion per year [3]. NASA estimated that the cost of corrosion to the USA was \$276 billion per year. This cost includes direct and indirect expenses associated with corrosion [4]. According to the report of the Japanese Ministry of Land, Infrastructure, Transport and Tourism, the damage types of steel bridges in Japan are shown in Figure 2 which proves that the corrosion is the most common damage type [5].

Many techniques already exist for corrosion monitoring. They range from direct mass loss measurements to characterization of the kinetics and thermodynamics involved. However there is always a need to develop more sensitive and convenient inspection systems to obtain more available 


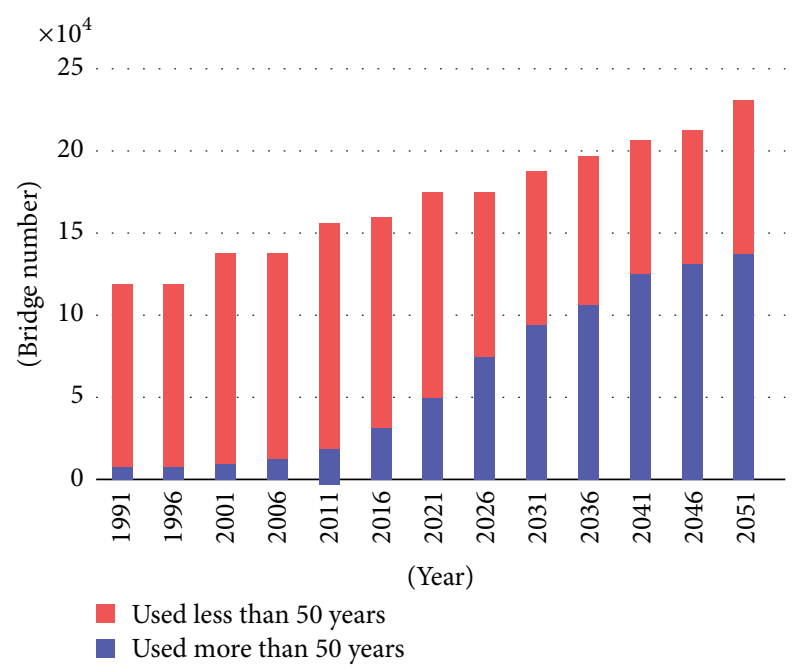

FIGURE 1: Numbers of steel bridges in Japan [23].

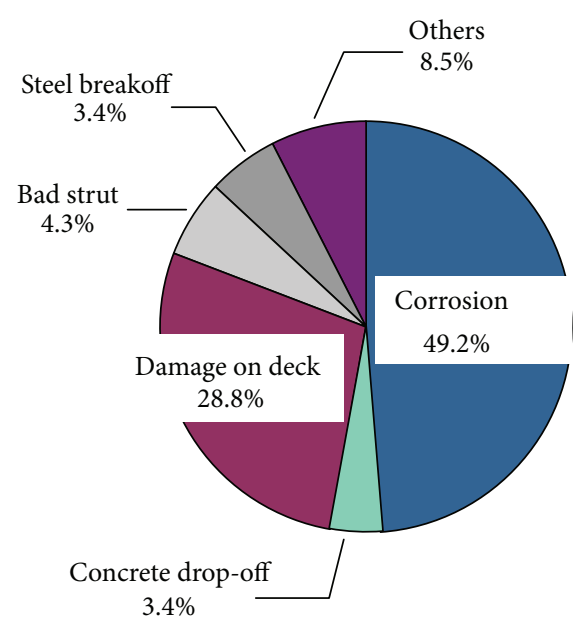

FIgURE 2: Corrosion is the most common type of deterioration of Japanese steel bridges.

information from the corroded steel bridges. Figure 3 shows two common traditional methods to inspect the steel bridge. One method uses scaffolds or ropes. Inspectors climb up to the bridge with scaffolds or ropes and inspect the bridge manually. The disadvantages of this method are obvious: it is inconvenient and dangerous to the inspectors because some bridges are high and large; inspection depends on the inspectors' experience and the results have subjective factors. The other traditional method is using a bucket truck or platform snooper. With the lifted equipment, inspectors can reach most areas of the bridge. This is a better way than using scaffold or ropes because the inspection cost can be cut and the safety of the inspectors is guaranteed to some degree. However, there are still disadvantages, such as the following: one lane of the road must be blocked by the inspection truck; inspectors have to stay in the lifting box for a long time according to the inspection time; it is still not safe for inspectors. Therefore, an automated inspection system for steel bridge inspection is strongly needed.
TABLE 1: Parameters of the robot.

\begin{tabular}{lc}
\hline Parameter & Value \\
\hline Length & $345(\mathrm{~mm})$ \\
Width & $210(\mathrm{~mm})$ \\
Height & $130(\mathrm{~mm})$ \\
Weight & $2.3 \mathrm{~kg}$ (without battery) \\
Drive & 2 motors $4 \mathrm{WD}$ \\
Operation & $2.4 \mathrm{GHz}$ remote control unit \\
\hline
\end{tabular}

\section{Robot Design}

A compact robot with 4 magnetic wheels for steel bridge inspection is developed. The locomotion concept is based on adapted lightweight magnetic wheel units with relatively high attractive force and friction force. The robot has the main advantages of being compact $(352 \times 215 \times$ $155 \mathrm{~mm})$, lightweight (2.38 kg without battery), and of a simple mechanical structure. It has only 4 active degrees of freedom (front wheel, rear wheel, an active servo actuator in the bending system, and a steering unit) and two passive degrees of freedom. It is not only able to climb vertical surfaces and inverted horizontal surfaces, but also able to pass complex combinations of $90^{\circ}$ convex and concave ferromagnetic corners with almost any inclination regarding gravity.

The prototype of the robot is shown in Figure 4. The robot consists of the bodywork made of alloy and carbon plates. There are two powerful brushed DC motors controlled by two commercial ESC (electronic speed control) units that are teleoperated by a commercial $2.4 \mathrm{GHz}$ wireless communication unit with PWM signals. Every wheel couple is actuated by one brushed DC motor coupled to a gearhead with reduction ratio of $44: 1$. The maximum speed of the robot can reach $0.32 \mathrm{~m} / \mathrm{s}$. Motors are mounted on the front axle and rear axle of the robot, respectively. The steering system, shock absorbers, and 4 magnetic wheels are also included. The robot itself is teleoperated by the inspector using a commercial $2.4 \mathrm{GHz}$ wireless communication unit that consists of the transmitter held by the inspector and receiver equipped on the robot. A FPGA evaluation board as a controller is used to actuate the servo actuator in the bending system by PWM signals, and it also would be used as a microcontroller unit for the sensing system. Figure 4 shows the prototype of the robot and Table 1 summarizes the basic parameters of the robot. In Figure 5, the experimental results validated that the robot had a good performance on locomotion on different surfaces of steel bridges.

\section{Magnetic Wheel Design}

Magnetic wheel design is the key point of the whole system. In this study, magnetic wheels are not only used as the adhesion device of the robot, but also regarded as an excitation source that provides the exciting magnetic field for nondestructive testing. It requires that the magnetic field induced by magnetic wheels be strong enough and keep consistent in direction and value when the wheel rotates. 

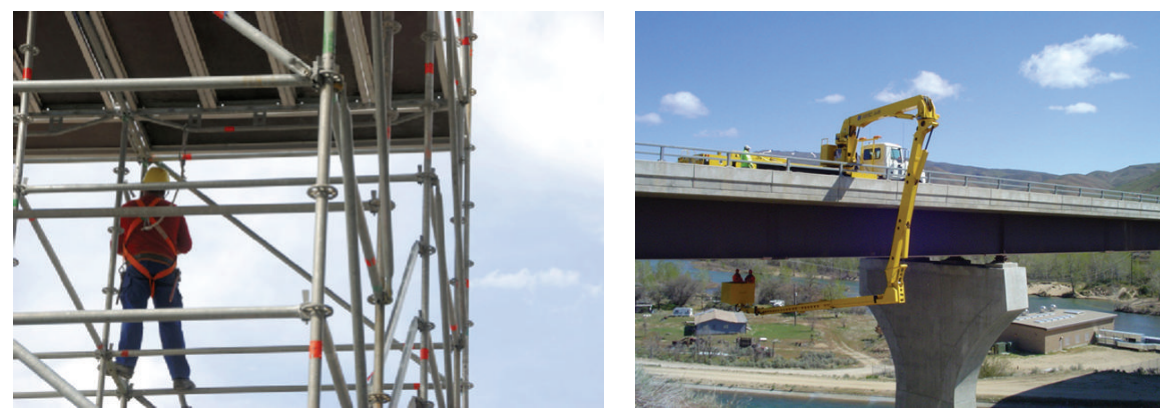

FIGURE 3: Two common ways to inspect bridges: scaffold and inspection truck with crane.

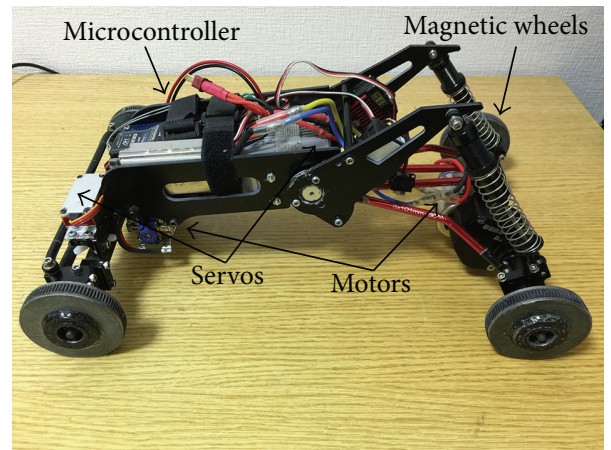

Figure 4: Prototype of the robot.
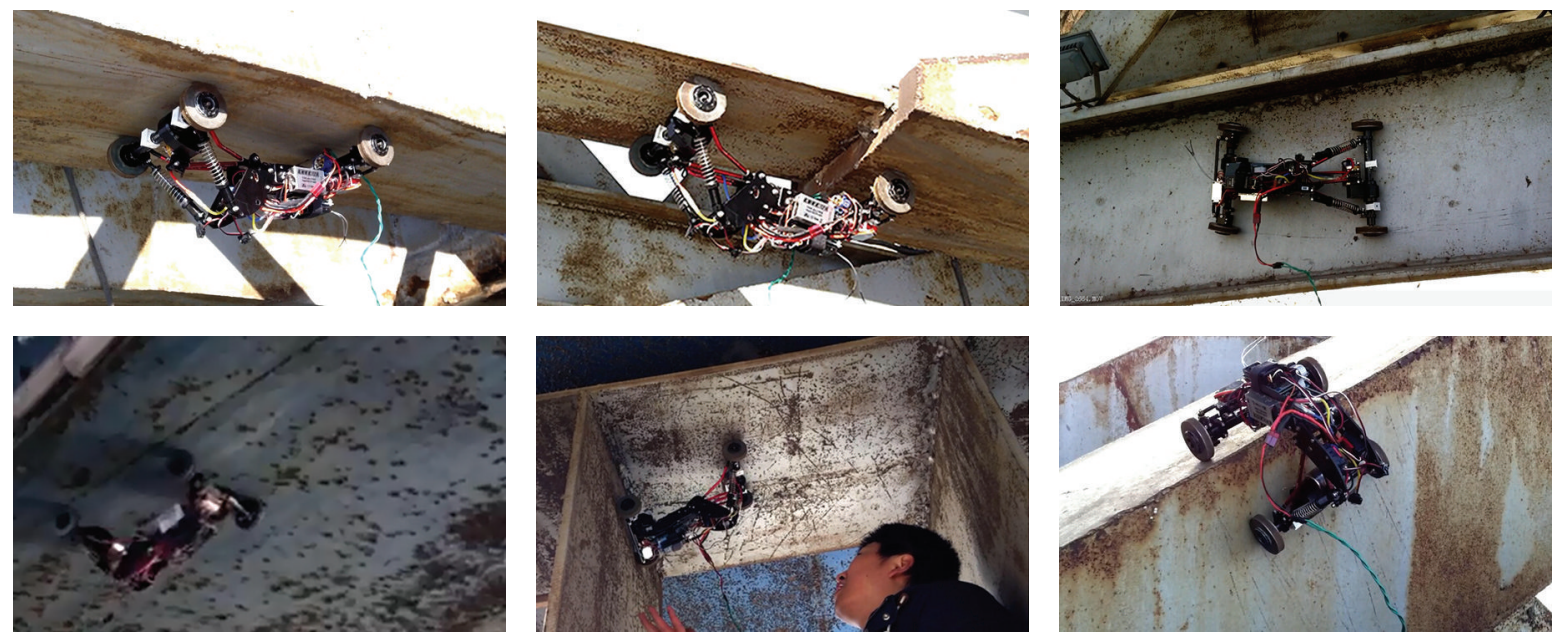

FIGURE 5: Locomotion of the robot in different parts of steel bridge.

It also has to provide enough attraction force and friction coefficient to make sure of the locomotion on the steel bridges. Furthermore, the size and the weight of the magnetic wheel have to be limited in order to keep the character compact and lightweight. Electromagnets and permanent magnets are considered to be used for the magnetic wheel design. The magnetic field generated by electromagnet can be switched on and off by controlling the current. However, constant power supply is needed to keep the adhesion force and it is difficult to be integrated into the magnetic wheel. For that reason, permanent magnet is considered as the best choice. Neodymium magnets are the strongest type of permanent magnet commercially available. Neodymium has higher magnetic field intensity to make sure of large magnetic force. Furthermore, it has high corrosion resistance, high hardness, and mechanical strength.

Figure 6 shows the prototype of magnetic wheel. The magnetic wheel consists of two ferromagnetic rings, a 


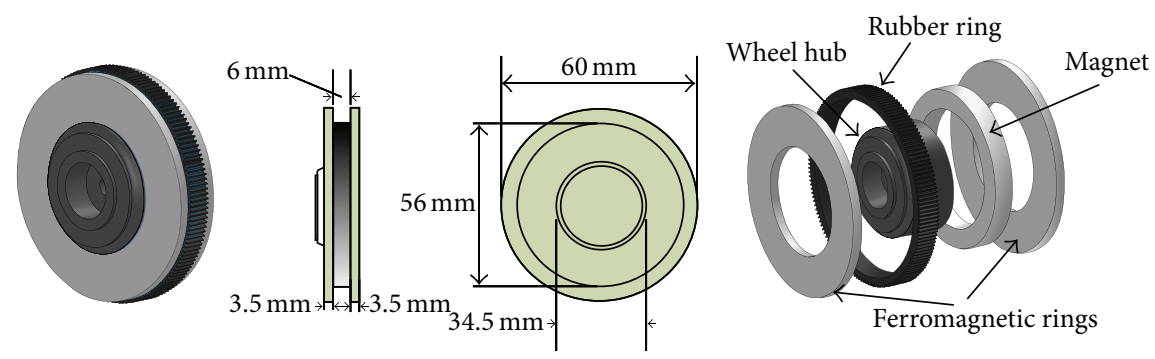

FIGURE 6: Magnetic wheel description.

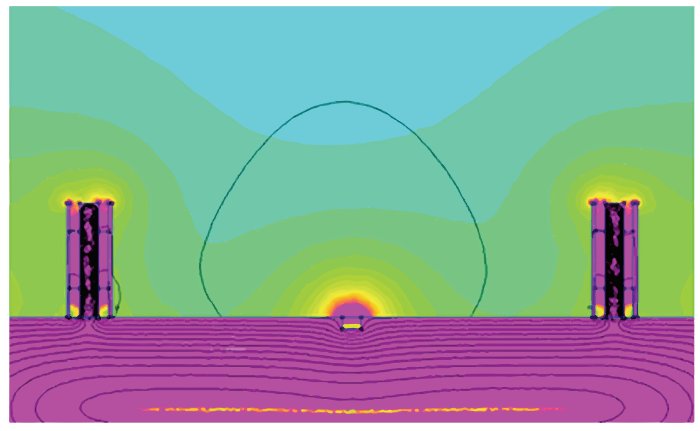

(a)

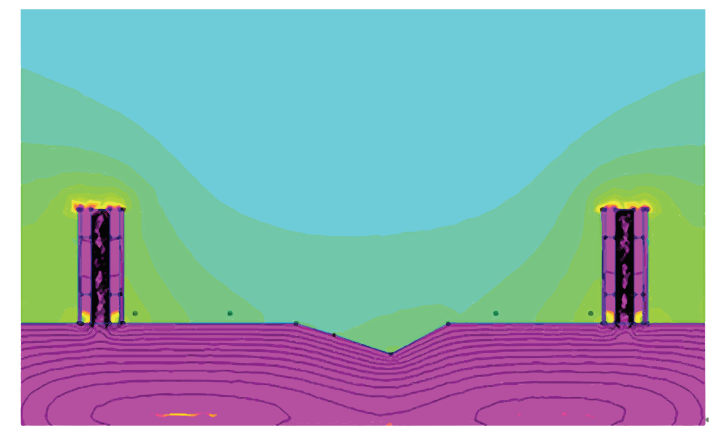

(b)

FIGURE 7: Unlike cracks, magnetic flux leakage method is not available in metal loss inspection. (a) A crack on the surface of the specimen. (b) A large metal loss on the surface of the specimen.

neodymium magnet ring, a wheel hub made of aluminum, and a rubber ring. Ferromagnetic rings are set on each side of the neodymium magnet ring in order to produce a magnetic flux through them and the specimen, which results in a strong attractive force to keep the robot on the surface of the specimen. The rubber ring is used to increase the friction coefficient, to enable the robot to overcome all kinds of obstacles. The high concentricity of magnet, wheel hub, and ferromagnetic rings are needed in order to provide constant magnetic flux density as an excitation source. The magnetic wheel is only $143 \mathrm{~g}$ in mass but even could provide the attraction force up to $4.4 \mathrm{kgf}$ and friction force up to $3.1 \mathrm{kgf}$ on the surface of the corroded steel board with a thickness of $8 \mathrm{~mm}$.

\section{Sensing System for Steel Bridge Inspection}

4.1. Limitation of Magnetic Flux Leakage Method and Eddy Current Method. The most commonly used NDT technologies for automation are visual inspection (camera, digital image processing, and so on), infrared thermography, magnetic testing (or magnetic flux leakage testing), acoustic emission, eddy current method, and so on. There have been some existing automated systems in which these NDT technologies are adopted to inspect the steel bridges [6-8]. However, from an ease of implementation perspective, some of NDT technologies normally used are not applicable for automation inspection. NDT sensing systems for automation inspection are assessed according to the following criteria: (1) size, weight, and manoeuvrability; (2) mode of operation; (3) type of power supply; (4) type of data collection; (5) level of operational accuracy; (6) overall suitability for automation. These criteria in turn dictate the robot specification such as weight, size, locomotion type, payload, and sensor type.

For ferromagnetic material NDT inspection, the magnetic flux leakage method and eddy current method are widely used, especially for fatigue crack and surface crack inspection. Both of the two methods have these advantages: (1) they have rapid testing; (2) they have compact size of sensing system; (3) surface preparation is not needed; (4) hidden defects and their size can be estimated; (5) they low cost and robust sensors.

Generally, the eddy current sensing system consists of excitation coils or permanent magnets and magnetic sensors such as Hall sensor, GMR (Giant Magnetoresistance) sensor, or gradient sensor [9-13]. In the presence of any discontinuity, such as a fatigue cracks, the linear induced current paths are distorted. The change of magnetic flux density induced by the eddy current is measured by the magnetic sensor. However, this method has proved to be not sensitive to the thickness of the corrosion layers. It provides little direct information on the corrosion process and rates of corrosion.

The magnetic flux leakage (MFL) method always needs to be used on the region of high saturation for the inspection of small metal loss or cracks. For that reason, it is limited to be used in the inspection of steel bridges with large and serious metal loss [12]. Figure 7 shows the simulations of the magnetic flux distribution when a fatigue crack and a metal loss exist on the surface of ferromagnetic material. It indicates that in the case of fatigue crack, the magnetic flux leakage 


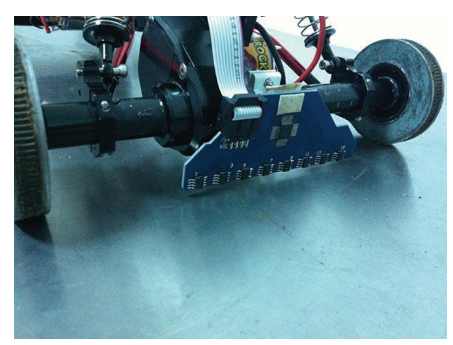

(a)

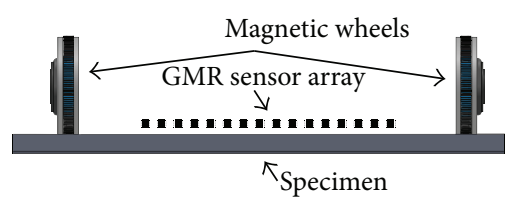

(b)

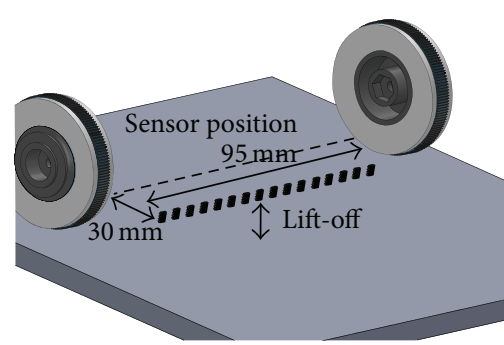

(c)

FIGURE 8: GMR sensor array design. (a) Prototype of the GMR sensor array. (b) Relative position of magnetic wheels GMR sensor array and specimen. (c) GMR sensor array position.

around the crack can be inspected by a magnetic sensor in an appropriate lift-off value. However, in the case of large metal loss corrosion, the magnetic flux leakage line cannot be inspected any more. Furthermore, for both eddy current method and MFL method, the lift-off noise introduced by varying the paint thicknesses, irregular specimen surface, or movement of sensors has a serious influence on the accuracy of the inspection. It greatly limits the application of eddy current method in quantitative nondestructive testing [14]. In some studies, the approaches to reduce lift-off noise in the eddy current method have been introduced [13-15]. However, steel bridges with levels of surface complexity are more difficult to be inspected. In addition, in some parts of the bridge, the materials with uneven surface are always used. It is difficult to inspect this kind of surface in eddy current method and MFL method. For example, checkered steel plates with rhombic shapes on the surface, which can be used as floor boards, factory stair boards, and deck boards are always used in steel bridge construction. As a result, a novel sensing system that is able to suppress lift-off noise and inspect uneven surfaces for automated metal losses inspection and early-stage corrosion inspection system is strongly needed.

4.2. Prototype of GMR Sensor Array. A schematic diagram of the sensor system is shown in Figure 8. It consists of two rear magnetic wheels and a GMR sensor array. Generally, considering the dimensions of the steel bridge, rapid automated detection is strongly needed. Linear sensor array has an inherent advantage for crack detection and tiny corrosion detection due to the fast moving speed of the inspection robot. In order to diminish the scanning time, 16channel GMR sensor array of NVE AA002 is used. Giant Magnetoresistance (GMR) is a device based on the giant magnetoresistivity phenomenon reported for the first time in 1988. It has the advantage of being robust in industrial noisy environments. GMR sensor is insensitive to magnetic fields perpendicular to the sensors' direction of sensitivity and the sensors' characteristic will not be disturbed if it is subject to strong magnetic fields. GMR sensor is used to measure the magnetic field density over a wide range. It is able to detect the magnetic field directly rather than the rate of change in magnetic field. It also has high sensitivity to small changes in magnetic field, which allows for accurate measurement of position or displacement in linear or rotational systems.
The design of the GMR sensor array was performed taking into account the size and the characteristics of the GMR sensor. NVE's GMR sensors have a primary axis of sensitivity. The flux concentrators on the sensor gather the magnetic flux along the axis and focus it at the GMR bridge resistors in the centre of the die. The sensor will have the greatest output signal when the magnetic field of interest is parallel to the flux concentrator axis [16].

Considering the geometry of the GMR sensor in a 3.00 $\times 3.00 \mathrm{~mm}$ SOIC8 package and the distance between two magnetic wheels, the centre distance of adjacent GMR sensor is $5 \mathrm{~mm}$ on each side of the PCB layer to ensure that the nondetection zone is eliminated. The advantages of using GMR sensor array are its simple mechanical structure and high reliability. Low power consumption is able to ensure the implementation of GMR sensor array design. Based on the experimental result, it can be indicated that power consumption of each GMR sensor is about $2 \mathrm{~mA}$ with $5.0 \mathrm{~V}$ supply voltage. This makes it possible to power all the sensors with one Li-Po battery at the same time without using multiplexer to shift the power to each sensor.

4.3. Characterization of the GMR Sensor. In many cases, GMR sensors make use of biasing magnetic fields to sense or create a pseudo zero field. Pseudo zero field is always used in the application of ferrous material inspection or detection where no magnetic field is present. Another case of biasing a GMR magnetic field is to provide a constant magnetic field in the sensitive direction of GMR sensor. Permanent magnet and current coils are generally applied for biasing GMR sensors part way up its output curve. In the literature [17], a GMR senor array was developed to inspect cracks on the surface of the specimen. A rectangular biasing coil mounted on the top of the GMR array was included as part of an eddy current probe in order to reflect the small differences between the static characteristics of each element of GMR sensor and the differences between the positions of the functioning point from one element to another. Elsewhere [18], a small permanent magnet is equipped on a fixed position near the GMR sensor to bias the sensor part way up its output curve.

In this paper, magnetic wheels are used to bias the GMR sensor array. Compared to the two biasing methods mentioned above, it conserves the energy and simplifies the hardware design. Generally, the magnetic characteristic of a 


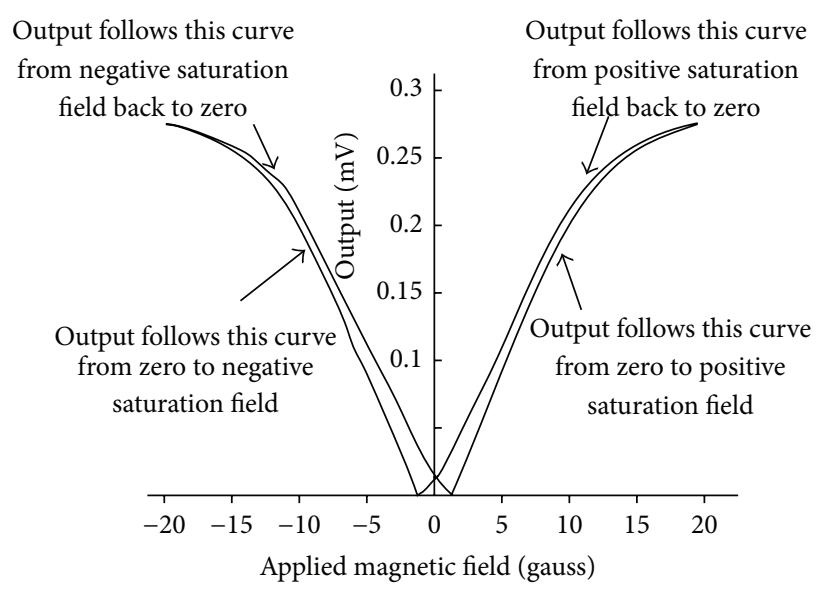

FIgURE 9: The output characteristic of GMR sensor AA002 [16].

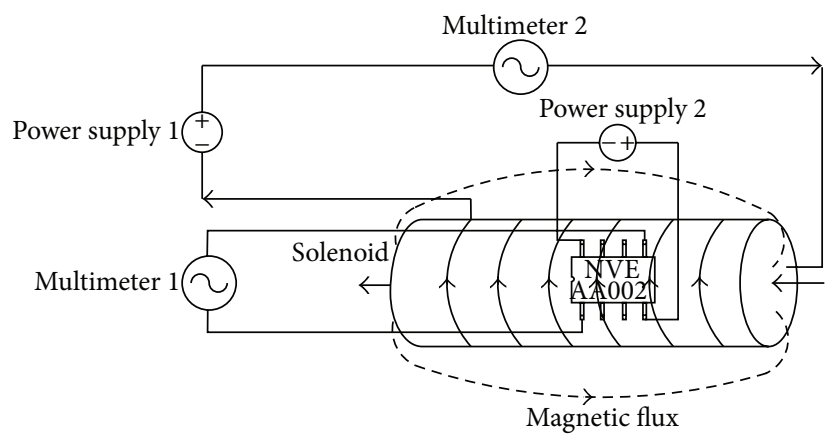

(a)

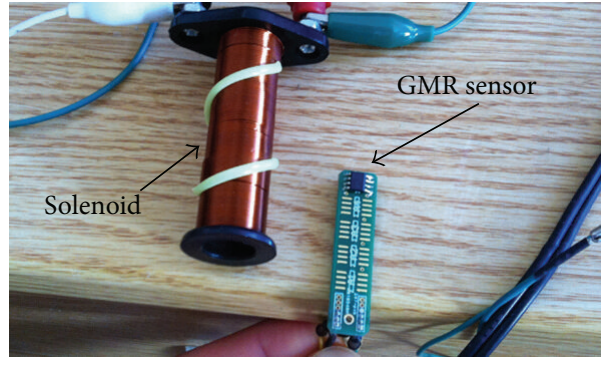

(b)

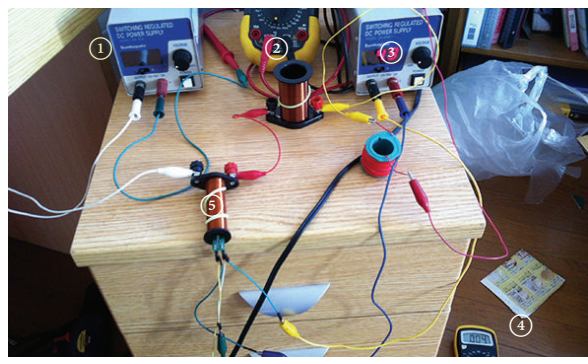

(c)

FIGURE 10: Experimental setup for AA002 characterization. (a) AA002 characterization hardware architecture. (b) GMR sensor was put into the centre of solenoid horizontally (c) (1): DC power number 1 for solenoid; (2), (4): multimeter; (3): DC power number 2 for GMR sensor; (5)-solenoid.

GMR sensor is shown in Figure 9. This characteristic was taken from AA002-02E GMR sensor with $5.0 \mathrm{~V}$ supplied to the bridge power terminals. There is a hysteresis effect in the output of the sensor as the magnetic field increases to a certain value. The initial output of the GMR sensor and sensitivity to the magnetic field are associated with magnetic biasing and power supply. These factors will deeply influence the output of the sensor. Therefore, it is necessary to characterize each GMR sensor before using it.

An experiment is implemented to characterize each GMR sensor. The schematic of experimental setup is presented in Figure 10. DC power number 1 and power number 2 are used to supply current to solenoid and voltage to GMR sensor, respectively. The value of current and the output of GMR sensor are measured by multimeters number 1 and number 2, respectively. GMR sensor is placed in the inner centre of the solenoid characterized by the following: length $L=57 \mathrm{~mm}$; diameter $D=16.7 \mathrm{~mm} ; N=240$ turns. According to Ampere's circuital law, the magnetic field inside an infinitely long solenoid is approximately considered to be homogeneous. It could be calculated approximately as

$$
B=\frac{\mu_{0} N I}{l},
$$




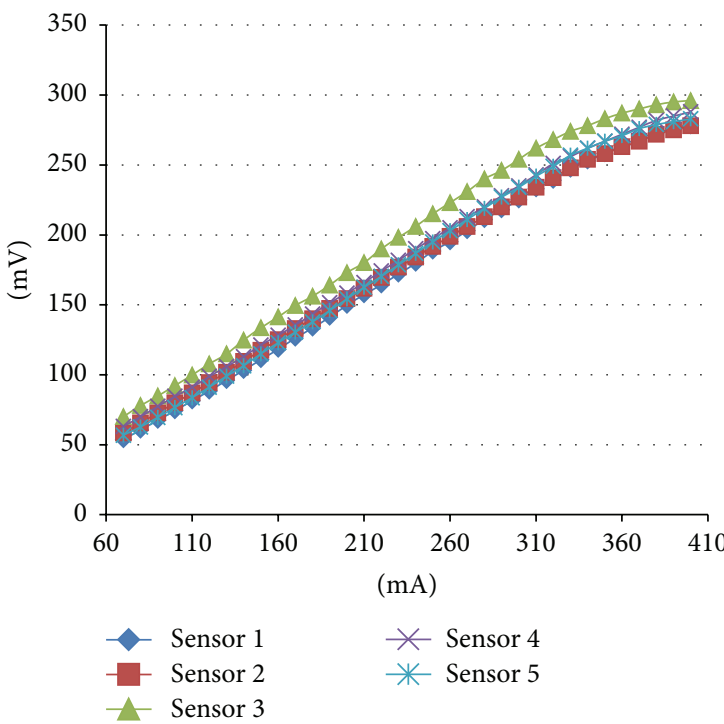

(a)

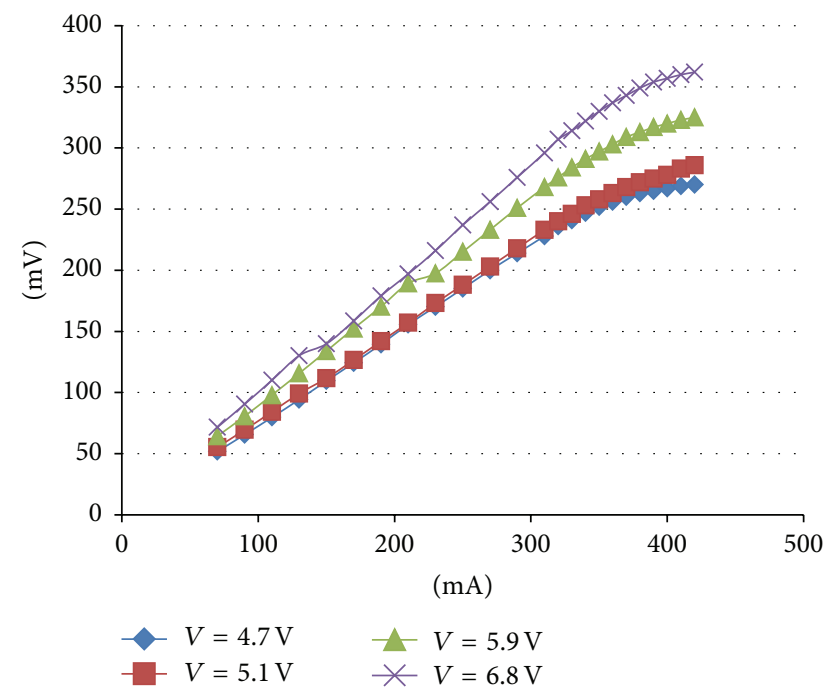

(b)

FIGURE 11: AA002 characterization. (a) The output curves of 5 GMR sensors with the same power supply. (b) The outputs of AA002 in different voltages of power supplies.

where $B$ is the magnetic field in the inner centre of the solenoid. $\mu_{0}$ is the magnetic constant. $I$ is the DC current passing solenoid. $N$ and $l$ are the turns of the wire and length of solenoid. It is obvious that magnetic field value is proportional to DC current. In the case of the output of power supply number 2 equaling $5.0 \mathrm{~V}, 5$ different GMR sensors named from S1 to S5 are placed in the centre of the solenoid, respectively, in order to observe sensitivities and hysteresis effect of each GMR sensor. As a result, the relationship between DC current and the output of the GMR sensor can be observed in Figure 11.

As can be observed in Figure 11(a), the initial outputs of GMR sensors are different to each other with the same $5.0 \mathrm{~V}$ power supply. However, the sensitivities of each GMR sensor are approximately the same. Because of the remanence effect of the GMR sensor that is made of magnetic material, the balance of Wheatstone bridge inside the GMR sensor could be changed by magnetic field nearby even if GMR sensors are powered off. As a result, the initial value would be changed when sensors are powered up. The solution to this problem is to bias the sensor element with an external magnetic field to make sure that the operating point of the sensor is on the linear portion of the characteristic curve shown in Figure 9.

On the other hand, the sensitivity of a GMR sensor would change with different voltages of power supply. Figure 11(b) shows the output of AA002 in different voltages of the power supply. It is indicated that as the increase of voltage of power, the sensitivity of the GMR sensor increases. It is important to ensure that the voltage of power supplied to each of GMR sensors keeps constant.

4.4. Lift-Off Noise Suppression. In eddy current-GMR sensor system, a planar excitation coil with unidirectional current excitation is used to generate a linear and uniform induced magnetic field. GMR sensor is in charge of measuring induced magnetic flux density. A scanning system is used to generate a C-scan image of the normal component of induced fields [19]. In the literature $[9,12,17,20]$, a linear GMR sensor array was considered to be used, which allows rapid scanning of an area for defects inspection in a single pass and with high testing resolution. In these studies, excitation coils had to be placed at a given height above the specimen to produce a constant magnetic flux density. Lift-off value of GMR was also kept constant to produce a C-scan image.

As stated in Section 4.1, both the MFL (magnetic flux leakage) method and eddy current method have a serious limitation of lift-off effect on steel bridge inspection. To use these two methods, lift-off value is required to keep constant and the surface of the sample must be flat enough. However, steel bridges always have irregular surface. Uneven surface would bring errors to the inspection results due to the change of lift-off value in the case that MFL method is used. Furthermore, in the early stage of corrosion, there is not any metal loss happening. As a result, it is impossible to detect early stage of corrosion using visual inspection or magnetic flux leakage method.

However, in the corroded place, the relative permeability would be changed. This change would lead to the change of magnetic flux density detected by the GMR sensor array. In this study, instead of current excitation coils, two magnetic wheels are used as an excitation source. As a result, in the area where sensors located, parallel magnetic field is obtained.

The finite element method (FEM) was employed to model the magnetic field distribution. Figures 12(a) and 12(b) show the magnetic field density distribution on the surface of the specimen and magnetic wheels. It validated that magnetic 


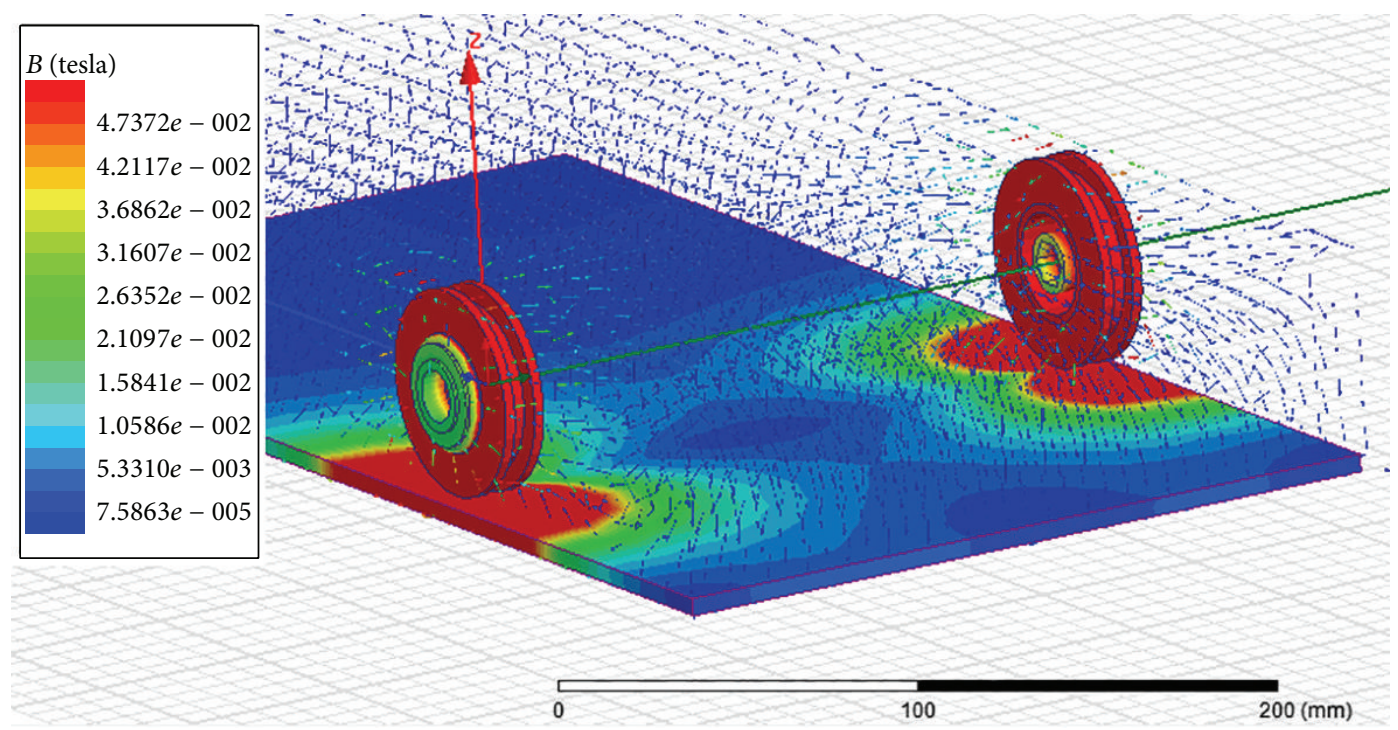

(a)

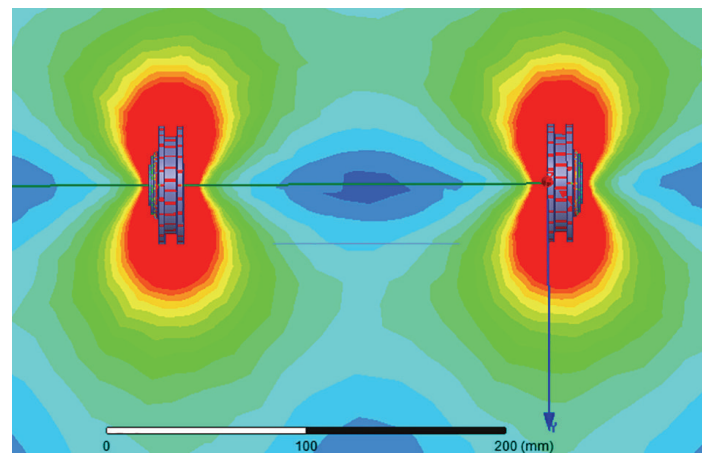

(b)

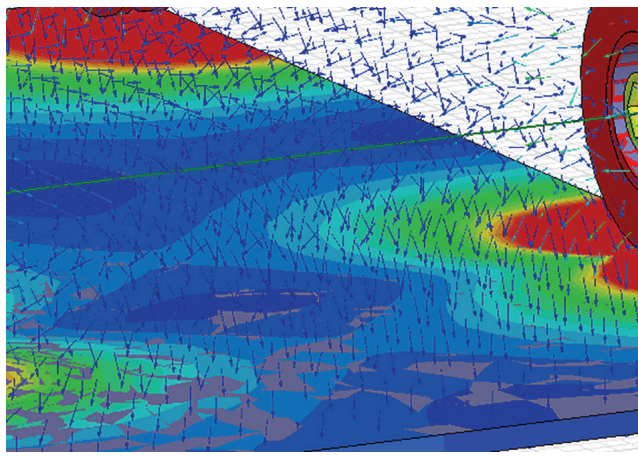

(c)

FIGURE 12: FEM model of magnetic wheels and specimen is built. (a) and (b) The density distribution of magnetic field on the surface of magnetic wheels and specimen. (c) The direction of induced magnetic flux line between two wheels.

field density is almost symmetric on the surface of the specimen without any defect. Figure 12(c) shows the spatial distribution of magnetic flux line. As shown in Figure 13, in the place where GMR sensor array located, the direction of magnetic field line is almost parallel and nearly perpendicular to the surface of the specimen. It is indicated that a small change in lift-off value does not have an obvious impact on the output of GMR sensor. This makes it possible to inspect the place where the surface is uneven on the steel bridge.

\section{Simulations and Experiments}

The simulation in Figure 14(a) shows the theoretical magnetic flux density where the GMR sensor array is located. The lift-off value of the GMR sensor array is set to $5 \mathrm{~mm}$. The result represents that in the nondefect region the distribution of magnetic flux density is parabolic and approximately symmetrical. However, there are still some asymmetrical places due to the tiny differences of the size and character of two magnetic wheels. This curve is considered as standard curve of magnetic flux density distribution in nondefect region of the ferromagnetic specimen. The simulation of the magnetic flux density in different lift-off is shown in Figure 14(b). The lift-off value is assigned as $2 \mathrm{~mm}, 5 \mathrm{~mm}$, and $10 \mathrm{~mm}$, respectively. The result shows that magnetic flux density keeps approximately the same value in the case that the lift-off value is set within $10 \mathrm{~mm}$. As the lift-off value continues to increase up to $10 \mathrm{~mm}$, the direction of magnetic flux density is no longer perpendicular to the surface of specimen. Displacement in vertical direction will cause a change of the output of GMR sensor array. It is indicated that within $10 \mathrm{~mm}$ the lift-off value variation basically does not have a serious impact on the output of GMR sensor array.

The magnetic flux density that the GMR sensor array detected can be shown in (2). B is the total magnetic flux density in the sensor position:

$$
B=B_{w}+B_{m}
$$




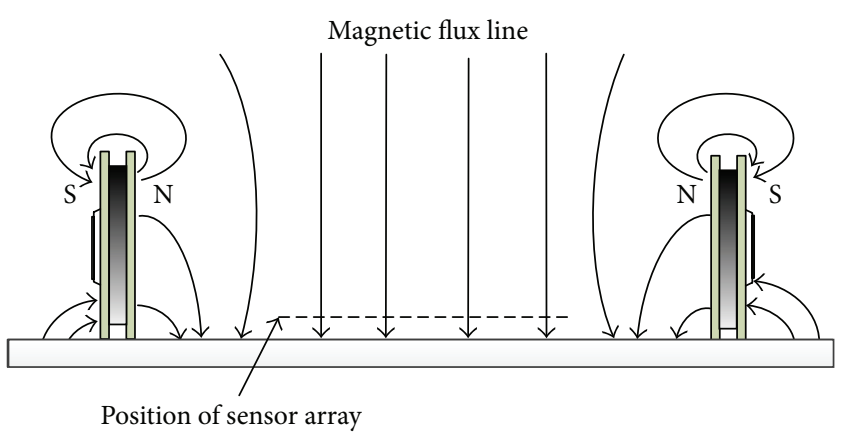

FIGURE 13: Distribution of magnetic flux line induced by magnetic wheels.

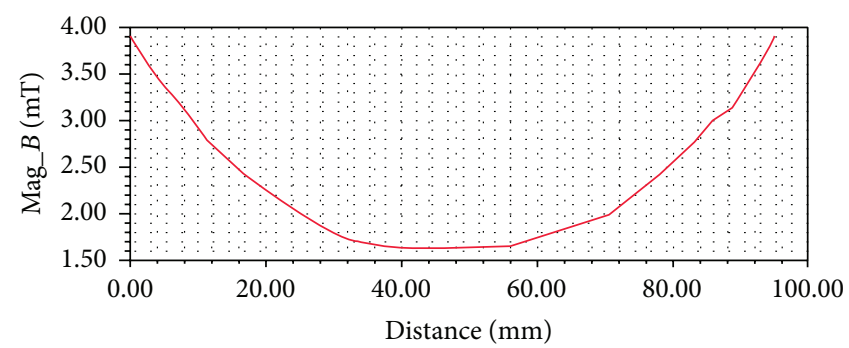

Lift-off $-5 \mathrm{~mm}$

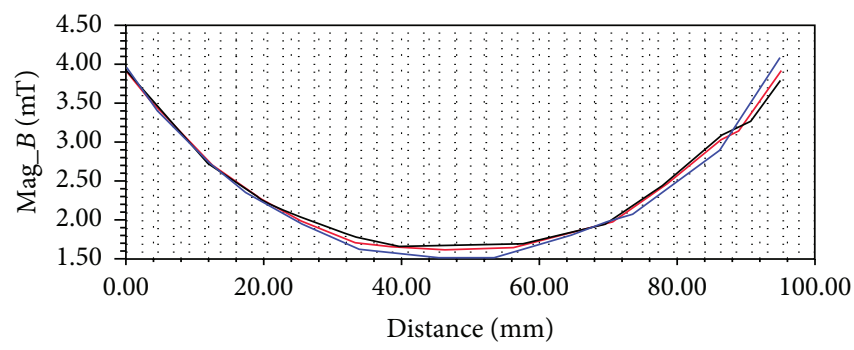

Lift-off

$-2 \mathrm{~mm}$

$-5 \mathrm{~mm}$

$-10 \mathrm{~mm}$

(a)

(b)

Figure 14: Simulation results of the magnetic field density where GMR sensor array is located. (a) Simulation result of the magnetic field density in the case that lift-off value is equal to $5 \mathrm{~mm}$. (b) Comparison of the simulation results in the case that lift-off value is equal to $2 \mathrm{~mm}$, $5 \mathrm{~mm}$, and $10 \mathrm{~mm}$, respectively.

$B_{w}$ is the magnetic flux density generated by magnetic wheels. $B_{m}$ is the magnetic flux density generated by the ferromagnetic material that is magnetized by magnetic wheels. As mentioned before, corrosion leads to a decrease of the relative permeability and the electrical conductivity of the material. As $B_{m}$ decreases, $B$ in (2) decreases.

Figure 15(a) shows a simulated circular corroded region with the diameter of $45 \mathrm{~mm}$ in the ferromagnetic specimen. As we know, the major component of corrosion is $\mathrm{Fe}_{2} \mathrm{O}_{3}$. According to study in the literature [21, 22], relative permeability and electrical conductivity are set to 1 and $100 \mathrm{~S} / \mathrm{m}$, respectively. Figure 16(a) shows the characteristic curve of magnetic flux density where GMR sensor array is located (black line) in the case of the lift-off equaling $5 \mathrm{~mm}$. Compared to the magnetic flux density of noncorrosion area (red line), a serious decrease of magnetic flux density can be observed in the corroded region due to the reduction of relative permeability and electrical conductivity.

Figure 15(b) shows a simulated crack defect whose width is $2 \mathrm{~mm}$. As the specimen magnetized by magnetic wheels, there is a distortion of magnetic flux leakage across the crack region in the specimen. Due to the reduction of magnetic permeability and cross-sectional area, magnetic flux lines leak out to the air from one side of the crack and come back to the specimen's surface again on the other side of the crack. This would lead to asymmetry of the magnetic flux density distribution between two magnetic wheels. As shown in Figure 17, bigger magnetic flux density can be detected in Areas 1 and 2 because of magnetic flux leakage. The simulation in Figure 16(b) validates the change of magnetic flux density, which increases on one side of the crack and decreases on the other side of the crack.

Figure 15(c) shows that the magnetic wheel is on the corroded region, whose relative permeability and electrical conductivity are set to 1 and $100 \mathrm{~S} / \mathrm{m}$, respectively [21, 22]. The corroded region is $80 \mathrm{~mm}$ long and $60 \mathrm{~mm}$ wide. As mentioned before, the corroded region that is in contact with the magnetic wheel has a large impact on the output of the GMR sensor array due to the decrease of the relative permeability. As Figure 16(c) shows, the left part of magnetic flux density curve almost overlaps with the standard curve because there is no corrosion under the right wheel. On the 


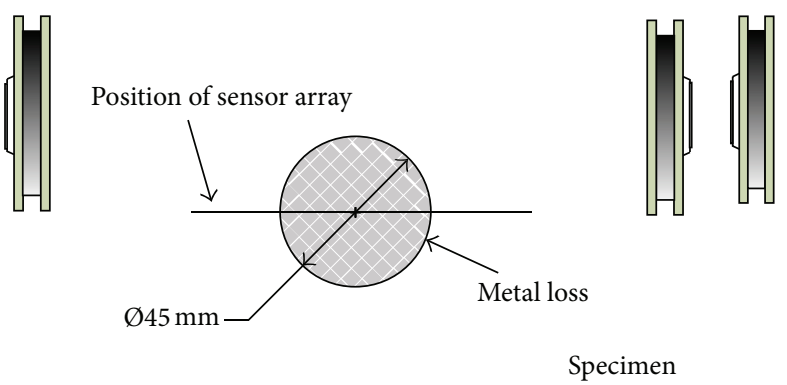

(a)

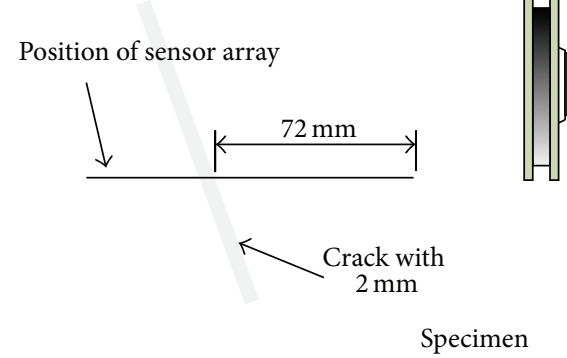

(b)

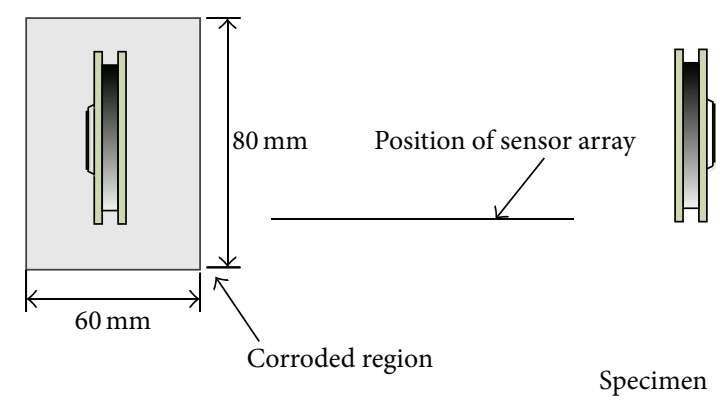

(c)

FIGURE 15: FEM models are built in the case that defects exist on the specimen. (a) Metal loss; (b) crack; (c) corrosion.
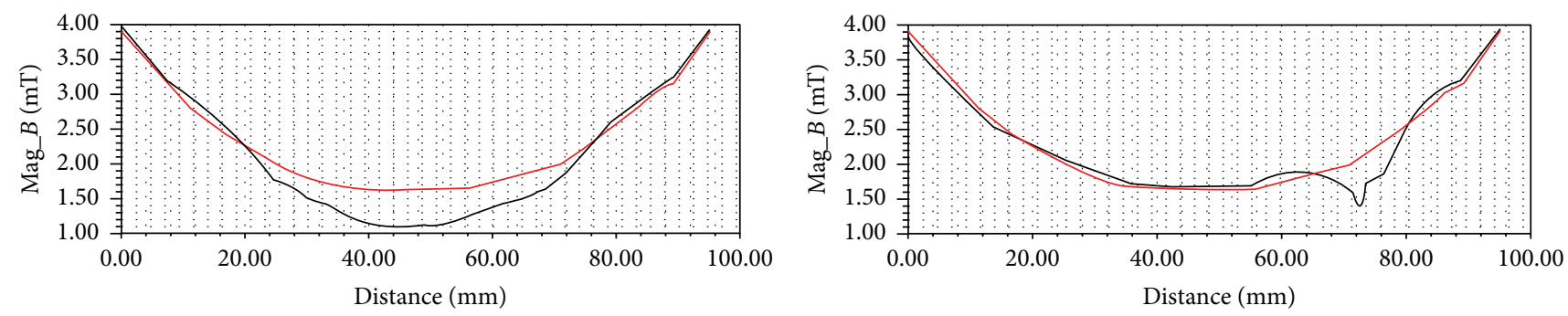

Lift-off $5 \mathrm{~mm}$

Without defect

Lift-off $5 \mathrm{~mm}$

With metal loss

- Without defect

With crack

(a)

(b)

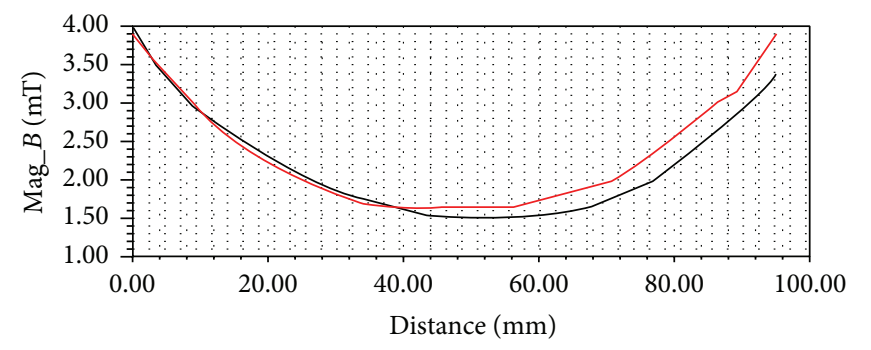

Lift-off $5 \mathrm{~mm}$

Without defect

- With corrosion

(c)

FIGURE 16: Simulation results of magnetic flux density in the position of GMR sensor array. (a) metal loss; (b) crack; (c) corrosion. 


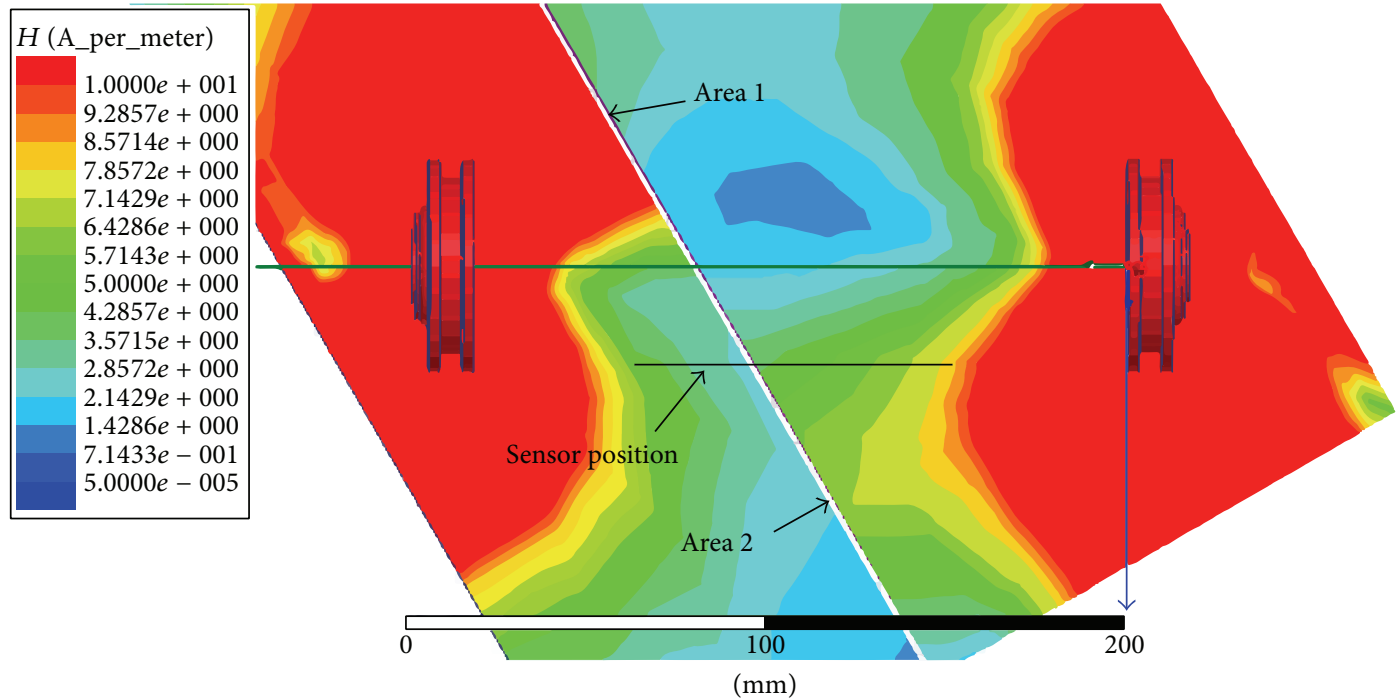

Figure 17: Distribution of magnetic flux line in the case that a crack exists on the specimen.

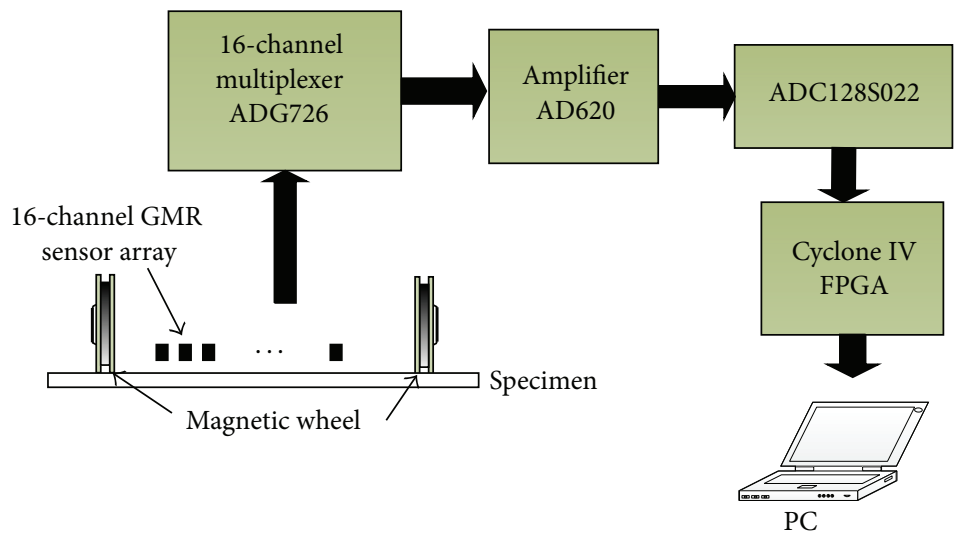

FIGURE 18: Experimental setup instrumentation.

other hand, compared to the standard curve, magnetic flux density in the region near the corrosion decreases as the black curve shows in Figure 16(c).

An experiment is implemented to validate the simulation result. Figure 18 shows experimental setup of the sensing system that consists of 16-channel GMR sensor array, high speed multiplexer ADG726, instrumentation amplifier AD620, A/D convertor ADC128S022, Altera Cyclone IV EP4CE22F17C6N FPGA, and PC. The GMR sensor array is powered up by $5.0 \mathrm{~V}$. 16 analog signals from GMR sensor array are amplified by an instrumentation amplifier AD620. Analog multiplexer ADG726 is in charge of switching the signals to instrumentation amplifier AD620 to make sure that it will not be the limiting determinant of the effective frame rate. To simplify the hardware design, only one ADC, which is embedded in the FPGA development board DE0-nano, is used to convert analogue signals into digital signals. The working process of data acquisition is shown in Figure 19.
During $2 \mathrm{us}$ time delay, the multiplexer shifts to the first channel; ADC begins to work and 100-time data acquisition is finished by ADC. The average value of 100 times data is calculated as the final output of this channel; the multiplexer switches to the next channel.

As mentioned before, the initial outputs of each GMR sensor are different to each other with the same power supply even if there is no magnetic field around it. The output of GMR sensor can be written as

$$
V=\alpha\left(B_{w}+B_{m}\right)+V_{0} .
$$

$\alpha$ is the sensitivity of the GMR sensor. It has been validated that the sensitivity of GMR sensor is constant with the same power supply. $B_{w}$ is the magnetic flux density generated by magnetic wheels. $B_{m}$ is the magnetic flux density generated by the ferromagnetic material, which is magnetized by magnetic wheels. $V_{0}$ is the initial output of GMR sensor. 


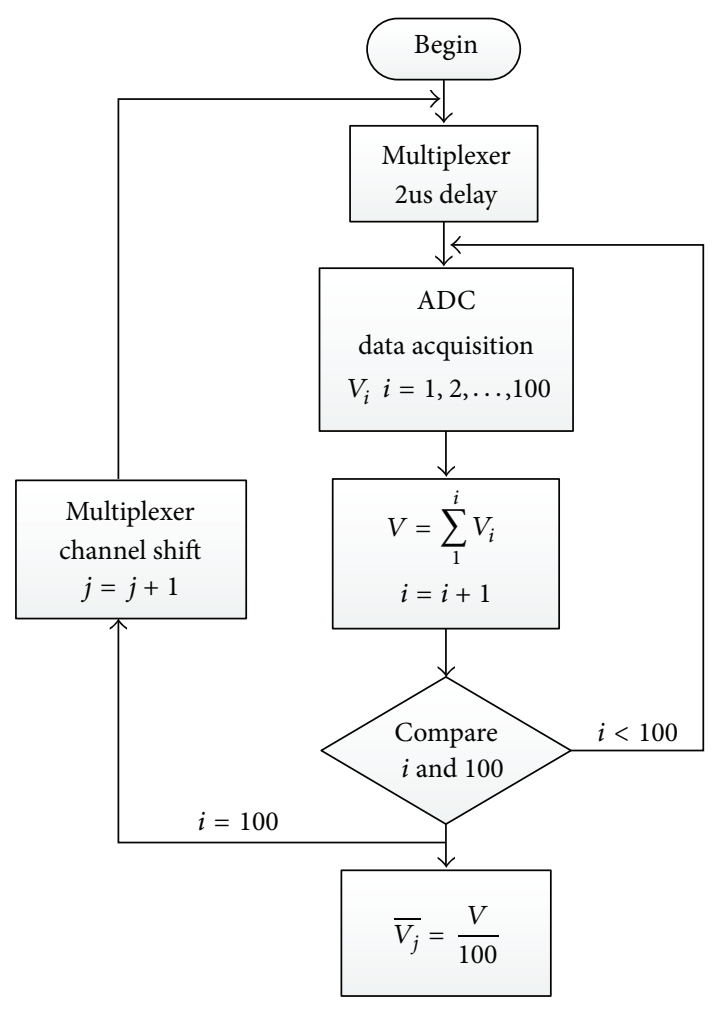

FIGURE 19: Process of data acquisition and processing.

Setting the power supply to $5 \mathrm{~V}$, in the region with defects, the output of the GMR sensor $V^{\prime}$ can be written as

$$
V^{\prime}=\alpha\left(B_{w}+B_{m}^{\prime}\right)+V_{0} .
$$

$B_{m}^{\prime}$ is the magnetic flux density generated by ferromagnetic material with defect. From (3) and (4), (5) is obtained:

$$
\Delta V=V^{\prime}-V=\alpha\left(B^{\prime}-B_{m}\right)=\alpha \Delta B_{m} .
$$

Defects in the ferromagnetic material cause $\Delta B_{m}$ which is proportional to $\Delta V$. As a result, the defects can be confirmed by comparing the change of the output of the GMR sensor.

A ferromagnetic plate $700 \mathrm{~mm}$ in length, $500 \mathrm{~mm}$ in width, and $6 \mathrm{~mm}$ in thickness is used as a test specimen. Three types of defects exist on the plate, as shown in Table 2. The metal loss is through the plate with approximately the length of $200 \mathrm{~mm}$ and width of $24 \mathrm{~mm}$. The length of the crack is approximately $150 \mathrm{~mm}$ and the width varies from 1.5 to $3 \mathrm{~mm}$. The character of the corrosion such as the relative permeability and the electrical conductivity is hard to estimate. Figure 20 shows the detection results of each kind of defect. Red line represents the standard output $V$ curve, the output of GMR sensor array on the region without any defect. In the defective area, $\Delta B_{m}$ in (5) increased due to the change of the relative permeability of the specimen. It leads to an obvious change of $\Delta V$.

In order to reflect defect information such as the boundary of the defect or corrosion degree, the sensing data need to be imaged. $\Delta B_{m}$ of each GMR sensor varies significantly from

\begin{tabular}{|c|c|c|}
\hline Type & Photography & Size \\
\hline Metal loss & & $\begin{array}{l}\text { Length: } 200 \mathrm{~mm} \\
\text { Width: } 24 \mathrm{~mm} \\
\text { Depth: } 6 \mathrm{~mm}\end{array}$ \\
\hline Crack & & $\begin{array}{l}\text { Length: } 150 \mathrm{~mm} \\
\text { Width: } 1.5-3 \mathrm{~mm} \\
\text { Depth: } 6 \mathrm{~mm}\end{array}$ \\
\hline Corrosion & & $\begin{array}{l}\text { Length: } 95 \mathrm{~mm} \\
\text { Width: } 65 \mathrm{~mm} \\
\text { Depth: unknown }\end{array}$ \\
\hline
\end{tabular}

TABLE 2: Parameters of the defects.

each other due to its relative position to the magnetic wheels. It is necessary to calibrate $\Delta V$ of each GMR sensor for a defect image. Relative calibrating coefficient of each GMR sensor $K_{i}$ $(i=1,2, \ldots, 16)$ is defined as

$$
K_{i}=\frac{V_{1}}{V_{i}} .
$$

$V_{i}(i=1,2, \ldots, 16)$ is the output of each GMR sensor of the standard output $V$ curve, which is shown in Figure 20(d). For instance, the relative calibrating coefficients of sensors number 1 and number 2 are

$$
\begin{aligned}
& K_{1}=\frac{3110}{V_{1}}=\frac{3110}{3110}=1, \\
& K_{2}=\frac{3110}{V_{2}}=\frac{3110}{2846}=1.0928 .
\end{aligned}
$$

$K_{i}$ is calculated and shown in Table 3. As a result, $\Delta V_{i}^{\prime}$ is defined as

$$
\Delta V_{i}^{\prime}=K_{i} \Delta V_{i}
$$

The value of $\Delta V_{i}^{\prime}$ is depicted in Figure 21. In metal loss detection experiment, the sensing system moved about $400 \mathrm{~mm}$. Figure 21(a) shows the values of $\Delta V_{i}^{\prime}$ obtained from the metal loss area. The size of the metal loss is about $260 \mathrm{~mm}$ $\times 40 \mathrm{~mm}$. The width of crack image shown in Figure 21(b) is about $20 \mathrm{~mm}$. Both of them are beyond the real size of the defects. It is because that distance between adjacent sensors gives an effect on the resolution of the sensing system.

In the corrosion detection experiment, the corroded area under the right magnetic wheel can be detected to some degree. It can be observed that the values of $\Delta V_{i}^{\prime}$ decrease on the right of the image in Figure 21(c). This appears to be 


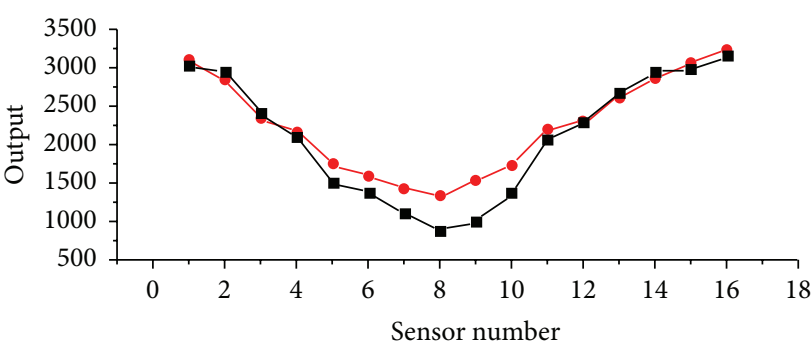

- Standard output $V$ curve-without defect

- Output $V^{\prime}$ curve-metal loss

(a)

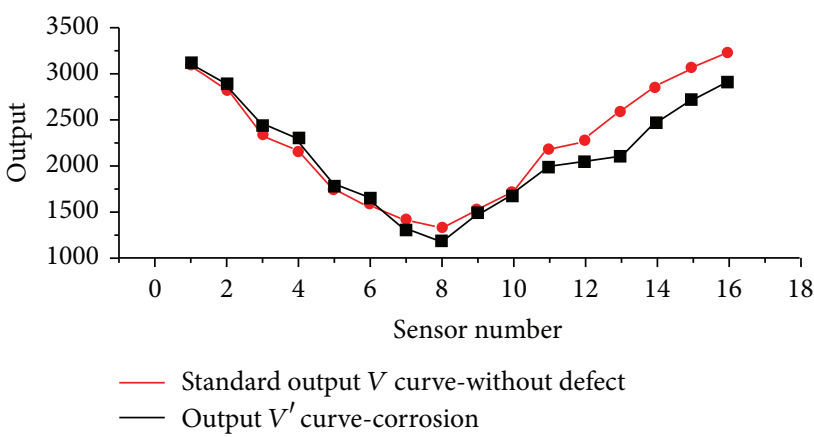

(c)

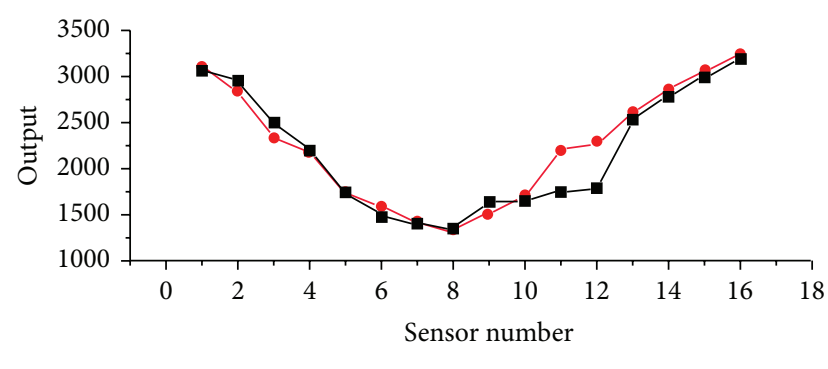

— Standard output $V$ curve-without defect

— Output $V^{\prime}$ curve-crack

(b)

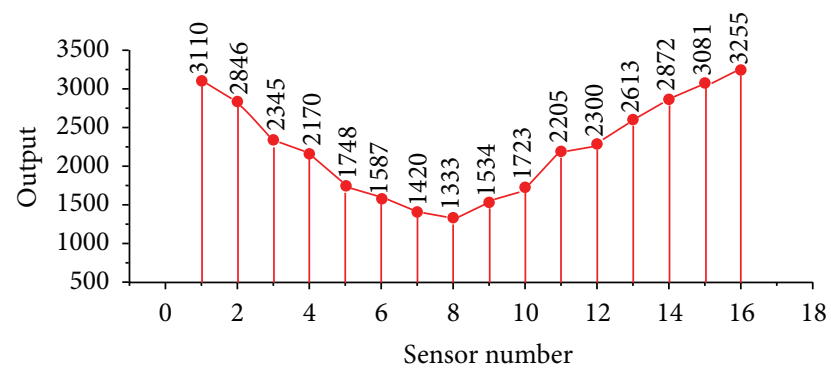

(d)

Figure 20: Voltage signals collected from data acquisition system. (a), (b), and (c) Showing the defect signals in the case of metal loss, crack, and corrosion. (d) The output signal of the standard output $V$ curve collected from data acquisition system.

TABLE 3: Relative calibrating coefficient $K_{i}$.

\begin{tabular}{cccccccccccccccc}
\hline$K_{1}$ & $K_{2}$ & $K_{3}$ & $K_{4}$ & $K_{5}$ & $K_{6}$ & $K_{7}$ & $K_{8}$ & $K_{9}$ & $K_{10}$ & $K_{11}$ & $K_{12}$ & $K_{13}$ & $K_{14}$ & $K_{15}$ & $K_{16}$ \\
\hline 1 & 1.0928 & 1.3262 & 1.4332 & 1.7792 & 1.9597 & 2.1901 & 2.3331 & 2.0156 & 1.805 & 1.4104 & 1.3522 & 1.1902 & 1.0829 & 1.0094 & 0.9555 \\
\hline
\end{tabular}

attributable to the reduction degree of relative permeability and electrical conductivity.

The experimental data partly verified the conclusions of simulation analysis. However, some differences between the experimental data and simulation results can be observed. Parameters set in simulations such as the relative permeability of the ferromagnetic materials and magnets are different from the ones used in the experiment. For example, in the simulation part, the relative permeability was set to 1 ; however, it is difficult to measure the actual relative permeability of the corroded area. Furthermore, the differences of shape and the size of the defect, the error of the distance within each sensor, the relative position from magnetic wheels, and sensor array also have an impact on the experimental results.

\section{Conclusions}

First of all, a climbing robot for steel bridge inspection was introduced. It has the ability to move on the surface of steel bridge and to negotiate obstacles. Then some of the NDT technologies used in ferromagnetic material inspection were introduced. As illustrated in Section 4.1, because of lift-off effect, eddy current method is difficult to be used for the inspection of steel bridge. MFL (magnetic flux leakage) is always used for crack inspection or tiny metal loss. However, it is difficult to inspect big metal loss under the paint or earlystage corrosion. To solve this problem, a novel sensing system with a GMR sensor array was developed. Relying on the interaction of induced magnetic fields generated by magnetic wheels of the climbing robot and without using current coils as excitation source, the GMR sensors array measures the differences of the magnetic field density between the defect region and the nondefect region. In the area sensor array located, the magnetic flux lines are parallel and almost perpendicular to the surface of the specimen. As a result, the change of the lift-off value in a certain range almost does not have an impact on the outputs of the GMR sensors. This method suppressed lift-off noise in metal loss inspection and corrosion inspection. Characterization of the GMR sensor was verified by using a solenoid with current. It is indicated that the initial outputs of each GMR sensor are different to each other and the sensitivity of the output of the GMR sensor is determined by the value of power supply. It is important to keep all the GMR sensors in the same power supply. Finally, three kinds of defects, metal loss, crack, and corrosion, were used to validate the feasibility of the sensing 


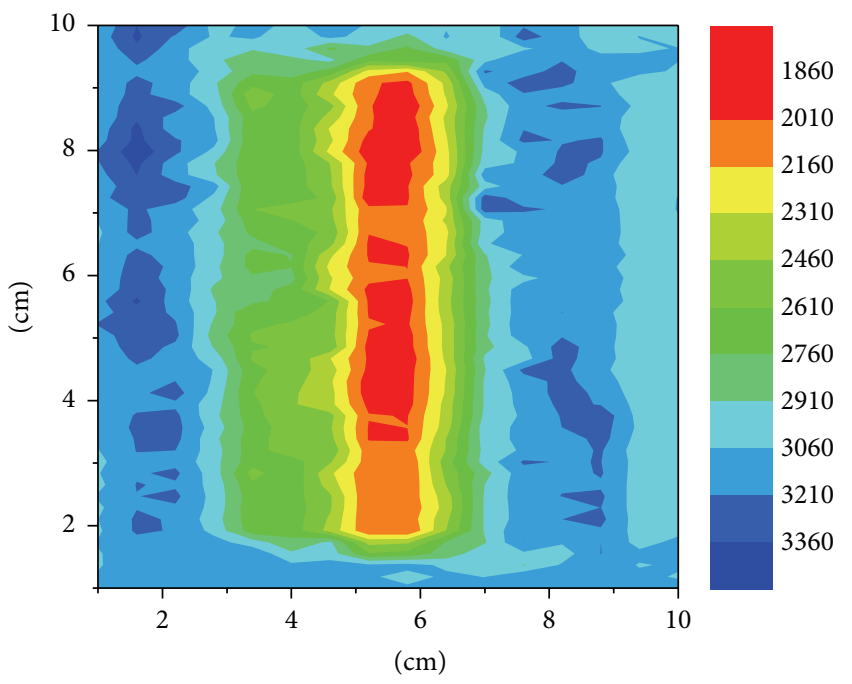

(a)

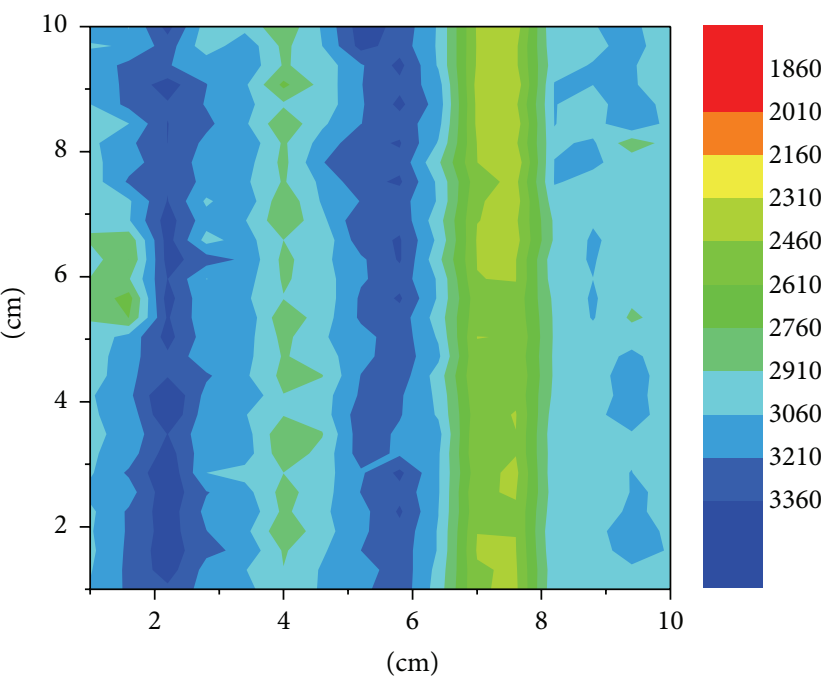

(b)

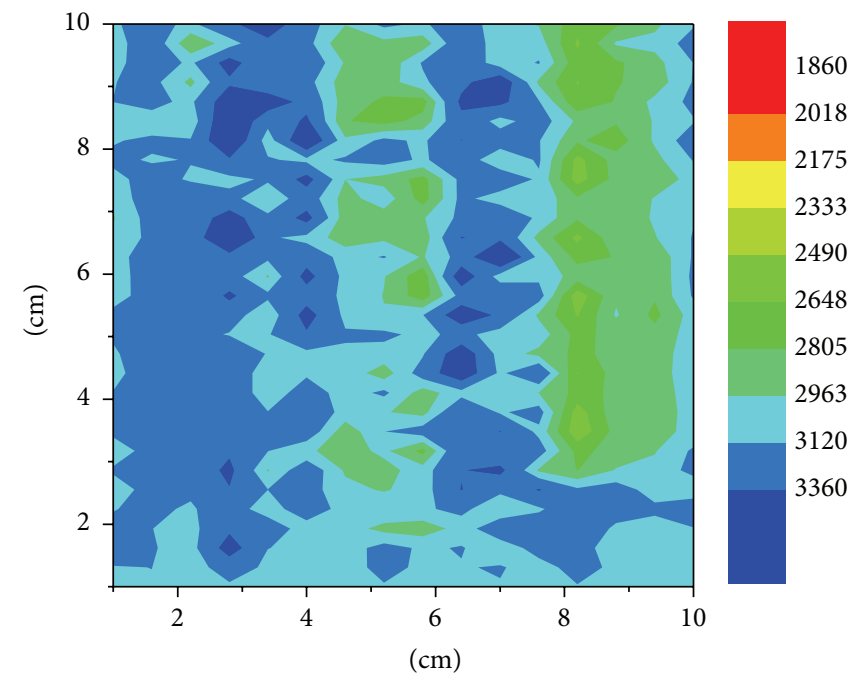

(c)

FIGURE 21: Defect images getting from the values of $\Delta V_{i}^{\prime}$ in the scanning area; (a) metal loss; (b) crack; (c) corrosion.

system. Simulations and experimental results showed that the sensing system was feasible to detect some kinds of defects including metal loss, cracks, and invisible corroded areas.

\section{Conflict of Interests}

The authors declare that there is no conflict of interests regarding the publication of this paper.

\section{References}

[1] I. Sugimoto, Y. Kobayashi, and A. Ichikawa, "Durability evaluation based on buckling characteristics of corroded steel deck girders," Quarterly Report of RTRI, vol. 47, no. 3, pp. 150-155, 2006.

[2] J. M. R. S. Appuhamy, M. Ohga, T. Kaita, P. Chun, and P. B. R. Dissanayake, "Estimation of corrosion-induced strength deterioration of steel bridge plates-an analytical method," Annual Research Journal of SLSAJ, vol. 11, pp. 19-25, 2001.
[3] M. Yunovich and N. G. Thompson, "Corrosion of highway bridges: economic impact and control methodologies," Concrete International, vol. 25, no. 1, pp. 52-57, 2003.

[4] Kennedy Space Center NASA, Corrosion technology laboratory, Electronically Corrosion Technology Laboratory, September 2014.

[5] September 2014, http://www.nilim.go.jp/english/eindex.htm.

[6] H. M. La, R. S. Lim, B. B. Basily et al., "Mechatronic systems design for an autonomous robotic system for high-efficiency bridge deck inspection and evaluation," IEEE/ASME Transactions on Mechatronics, vol. 18, no. 6, pp. 1655-1664, 2013.

[7] H. Leon-Rodriguez, S. Hussain, and T. Sattar, "A compact wall-climbing and surface adaptation robot for non-destructive testing," in Proceedings of the 12th International Conference on Control, Automation and Systems (ICCAS '12), pp. 404-409, IEEE, JeJu Island, South Korean, October 2012.

[8] A. Leibbrandt, G. Caprari, U. Angst, R. Y. Siegwart, R. J. Flatt, and B. Elsener, "Climbing robot for corrosion monitoring of 
reinforced concrete structures," in Proceedings of the 2nd International Conference on Applied Robotics for the Power Industry (CARPI '12), pp. 10-15, IEEE, Zürich, Switzerland, September 2012.

[9] M. M. Tehranchi, M. Ranjbaran, and H. Eftekhari, "Double core giant magneto-impedance sensors for the inspection of magnetic flux leakage from metal surface cracks," Sensors and Actuators, A: Physical, vol. 170, no. 1-2, pp. 55-61, 2011.

[10] B. Wincheski, F. Yu, J. Simpon, P. Williams, and K. Rackow, "Development of SDT sensor based eddy current probe for detection of deep fatigue cracks in multi-layer structure," NDT and E International, vol. 43, no. 8, pp. 718-725, 2010.

[11] G. Yang, Z. Zeng, Y. Deng et al., "3D EC-GMR sensor system for detection of subsurface defects at steel fastener sites," NDT and E International, vol. 50, pp. 20-28, 2012.

[12] N. B. S. Gloria, M. C. L. Areiza, I. V. J. Miranda, and J. M. A. Rebello, "Development of a magnetic sensor for detection and sizing of internal pipeline corrosion defects," NDT and E International, vol. 42, no. 8, pp. 669-677, 2009.

[13] W. Li, X. Yuan, G. Chen, X. Yin, and J. Ge, "A feed-through ACFM probe with sensor array for pipe string cracks inspection," NDT \& E International, vol. 67, pp. 17-23, 2014.

[14] Y. Yu, Y. Yan, F. Wang, G. Tian, and D. Zhang, "An approach to reduce lift-off noise in pulsed eddy current nondestructive technology," NDT and E International, vol. 63, pp. 1-6, 2014.

[15] G. Y. Tian and A. Sophian, "Reduction of lift-off effects for pulsed eddy current NDT, NDT \& E International, vol. 38, no. 4, pp. 319-324, 2005.

[16] NVE GMR Magnetic Sensors Application Notes, 2014, http:// www.nve.com/SensorApps.php.

[17] O. Postolache, A. L. Ribeiro, and H. G. Ramos, "GMR array uniform eddy current probe for defect detection in conductive specimens," Measurement, vol. 46, no. 10, pp. 4369-4378, 2013.

[18] J. E. Simms, H. H. Bennett Jr., R. A. Goodson et al., The Detection and Discrimination of Small Munitions using Giant Magnetoresistive (OMR) Sensors, US Army Corps of Engineers-Engineer Research \& Development Center, Vicksburg, Miss, USA, 2010.

[19] G. Yang, G. Dib, L. Udpa, A. Tamburrino, and S. S. Udpa, "Rotating field EC-GMR sensor for crack detection at fastener site in layered structures," IEEE Sensors Journal, vol. 15, no. 1, pp. 463-470, 2015.

[20] A. Yashan, W. Bisle, and T. Meier, "Inspection of hidden defects in metal-metal joints of aircraft structures using eddy current technique with GMR sensor array," in Proceedings of the 9th European NDT Conference, Berlin, Germany, September 2006.

[21] M. Hotta, M. Hayashi, and K. Nagata, "Complex permittivity and permeability of $\alpha-\mathrm{Fe}_{2} \mathrm{O}_{3}$ and $\mathrm{Fe}_{1-x} \mathrm{O}$ powders in the microwave frequency range between 0.2 and $13.5 \mathrm{GHz}$, ISIJ International, vol. 50, no. 10, pp. 1514-1516, 2010.

[22] W. M. Telford, L. P. Geldart, and R. E. Sheriff, Applied Geophysics, vol. 1, Cambridge University Press, 1990.

[23] R. Wang and Y. Kawamura, "A magnetic climbing robot for steel bridge inspection," in Proceedings of the 11th World Congress on Intelligent Control and Automation (WCICA '14), pp. 3303-3308, Shenyang, China, June 2014. 


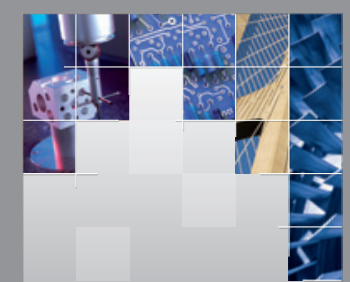

\section{Enfincering}
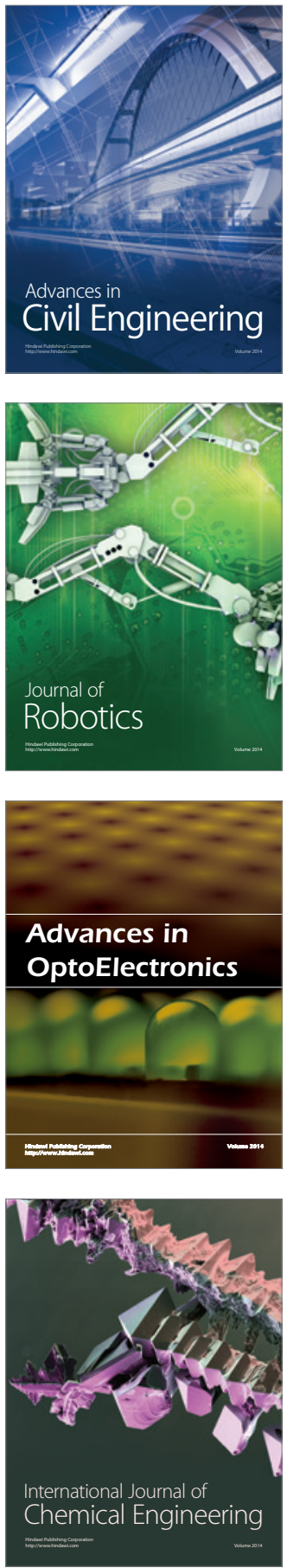

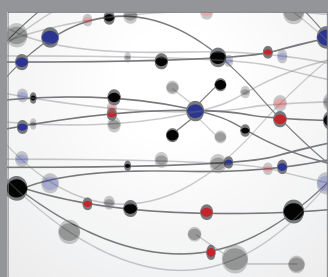

The Scientific World Journal

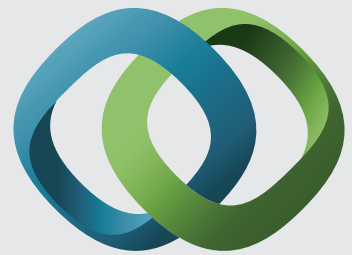

\section{Hindawi}

Submit your manuscripts at

http://www.hindawi.com
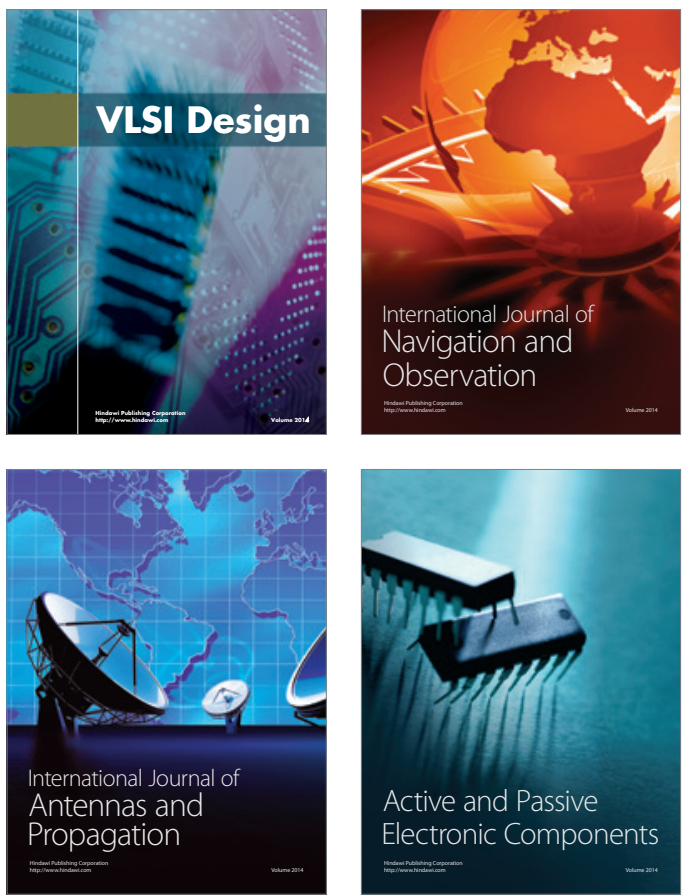
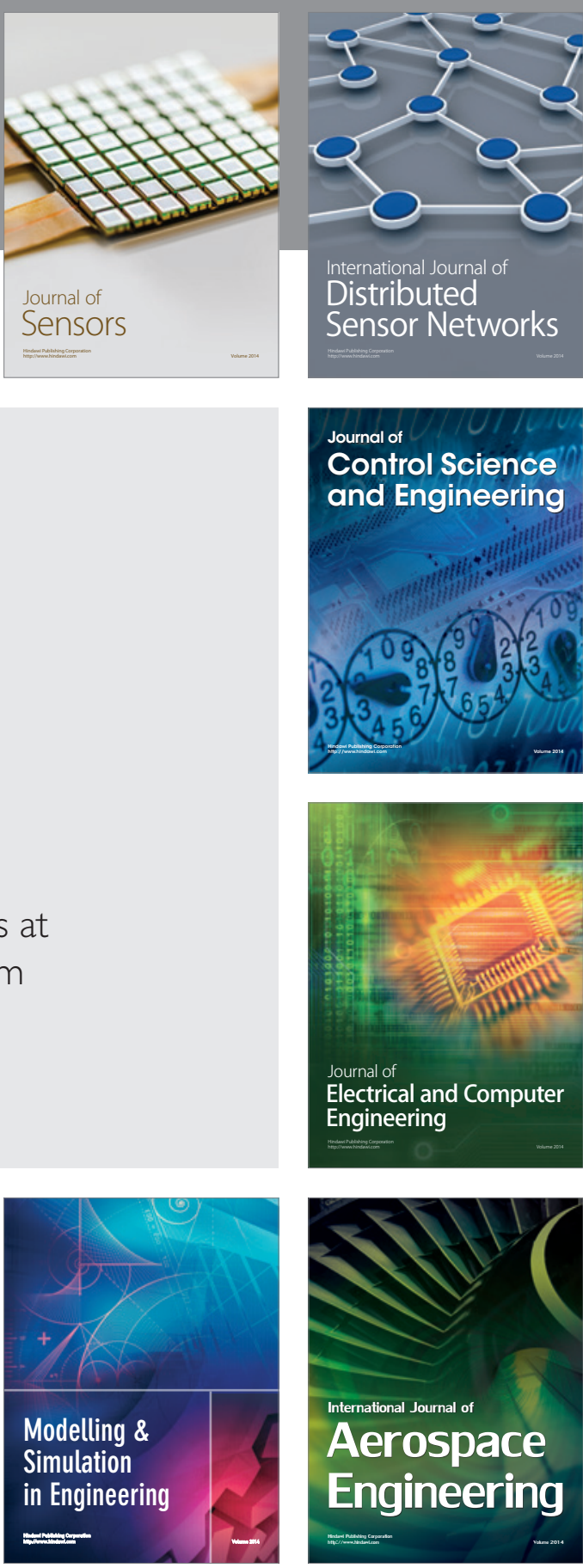

International Journal of

Distributed

Sensor Networks

Journal of

Control Science

and Engineering
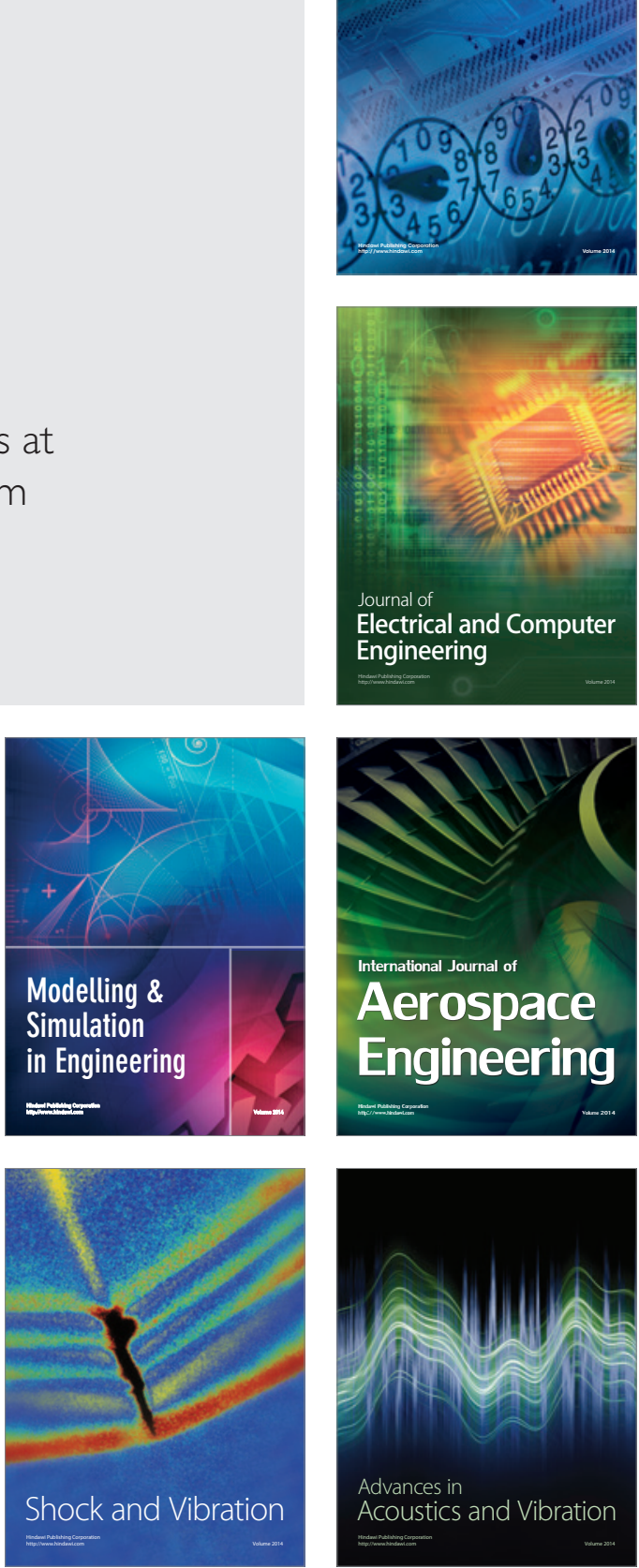